Estudios Románicos, Volumen 29, 2020, pp. 55-72

ISSN: 0210-4911

eISSN: $1989-614 \mathrm{X}$

DOI: https://doi.org/10.6018/ER.420781

\title{
LE VOYAGE COMME PALIMPSESTE IDENTITAIRE DANS GRANDE SECTION, LE PREMIER ROMAN D'HADIA DECHARRIÈRE
}

(The travel as an identity palimpsest in Grand Section, Hadia Decharrière's first novel)

\author{
Ana Belén Soto* \\ Universidad Autónoma de Madrid
}

\begin{abstract}
In the current context, travel and movement play an essential role in defining geopolitical boundaries as well as identity. In this sense, it is worth highlighting how the literary mosaic of contemporary Francophone xenographs reflects the imprint of current migratory processes that draw a new promenade and landscape in Romanesque literature. In this article, we propose to analyse Grande Section, Hadia Decharrière's first novel, with the aim of illustrating the preponderance of autofictional writing in the literary and cited field. Also, this novel will allow us to address the different meanings that the topic of the trip acquires in its scriptural project. This theme, initially linked to geographical displacement, will allow us to outline the consequences of this identity uprooting whit ontological significance.
\end{abstract}

Keywords: Hadia Decharrière; Travel; Autofiction; Francophone literature; Identity; Women's writen.

Resumen: En el contexto actual, el viaje y el movimiento desempeñan un papel esencial en la definición de fronteras geopolíticas a la vez que identitarias. En este sentido, cabe poner de relieve cómo el mosaico literario de las xenografías francófonas contemporáneas refleja la impronta de los procesos migratorios actuales que dibujan un nuevo paseo y paisaje en las letras románicas. En el presente artículo, nos proponemos analizar Grande Section, la primera novela de Hadia Decharrière, con el objetivo de ilustrar la preponderancia de la escritura autoficcional en el ámbito literario ya citado. Asimismo, esta novela nos permitirá abordar los matices particulares que la temática del viaje adquiere en su proyecto

* Dirección para correspondencia: Ana Belén Soto. Dpto. de Filología Francesa. Facultad de Filosofía y Letras. Universidad Autónoma de Madrid. Campus de Cantoblanco (anabelen.soto@uam.es). 
escriptural. Su temática, inicialmente ligada al desplazamiento geográfico, nos permitirá esbozar las consecuencias de este desarraigo identitario con significado ontológico.

Palabras clave: Hadia Decharrière; Viaje; Autoficción; Literatura francófona; Identidad; Escritoras.

\section{Introduction ${ }^{1}$}

Autrefois, les hommes avaient le sentiment d'être éphémères dans un monde immuable $[\ldots]$.

« De mémoire de rose, on n'a jamais vu mourir un jardinier », soupiraient les philosophes français des Lumières en songeant à l'ordre social et à la monarchie de leur propre pays. Aujourd'hui, les roses pensantes que nous sommes vivent de plus en plus longtemps, et les jardiniers meurent. En l'espace d'une vie, on a le temps de voir disparaître des pays, des empires, des peuples, des langues, des civilisations.

L’humanité se métamorphose sous nos yeux (Maalouf 2019 : 11-12).

En toile de fond d'une telle réflexion, Amin Maalouf met en évidence le caractère changeant, muable et instable du monde qui nous entoure. De nos jours, l'hybridation, la prolifération et l'accélération font également partie d'un quotidien marqué par les flux culturels globaux (Appadurai 1996) ainsi que par les déplacements multiformes. Les raisons qui poussent l'homme à partir sont ainsi diverses, elle peuvent répondre à un besoin socio-professionnel, à une volonté personnelle ou à une fuite suite à des événements politiques, par exemple. Pour Filippo Zanghi (2014 : 26) « voyager, voire se promener, c'est sortir de l'ordinaire et jeter un regard neuf sur le monde », le voyage est également " un moyen de connaître l'Ailleurs et l'Autre, d'échaper au quotidien » (Tverdota 1994 : 89) et le voyageur évoque ainsi le visiteur qui découvre de nouveaux horizonts géopolitiques et qui éprouve une expérience sensorielle nouvelle, à la fois qu'il illustre le regard nouveau, parfois même sur l'univers connu. Force est de constater que le voyage, devenu un acte quotidien dès l'aube du XXI siècle, implique une multiplicité de perspectives intimement liées à la nature même du déplacement. Et, ce rapport à la mouvance relève d'un certain nomadisme inhèrent au sujet contemporain qui désormais, toujours en transit ou en déplacement, se voit définit à travers les " nouvelles technologies et [les] nouvelles vitesses [qui] comportent toutes une référence directe à l'espace et à la dimension du passage et du mouvement » (Calefato 2018 : 148).

C'est dans ce contexte que la politologue Catherine Wihtol de Wenden (2013:44) constate la multiculturalité des sociétés contemporaines et prône une réflexion profonde sur les " nouveaux visages de la mobilité et de la coprésence, ici et là-bas ». Les flux migratoires contribuent, par conséquent, à l'évolution des paradigmes nationaux et acquièrent un rôle majeur dans les sociétés contemporaines composées désormais de sujets aux identités multiples. Les personnes en déplacement, soit en transit soit

1 Ce travail s'inscrit dans le cadre des objectifs du projet de recherche $\mathrm{I}+\mathrm{D}+\mathrm{i}$ du "Ministerio de Ciencia e Innovación" (référence: PID 2019-104520GB-I00). 
de manière permanente, illustrent ainsi une traversée identitaire vers la découverte de l'autre, mais aussi vers la découverte d'une identité personnelle dorénavant empreinte de l'entre-deux. Les notions telles que déracinement, dépaysement, déterritorialisation ou encore reterritorialisation permettent, par conséquent, de penser la mosaïque sociale contemporaine en termes d'inclusion.

À ce stade de la réflexion, il convient de signaler que l'activité intellectuelle et académique se nourrit également des réflexions de ces nouveaux intervenants de la société. En France, c'est ainsi qu'Amin Maalouf, né à Beirut en 1949, occupe un siège à l'Académie Française ; que Tveztan Todorov (1939-2017), d'origine bulgare, a obtenu en 2001 le Prix de philosophie décerné par l'Académie Française et connu sous le nom de La Bruyère ; et, que bon nombre d'écrivains en herbe comme Maryam Madjidi (née à Téhéran en 1980) sont lauréats des prix de renom tels que le Prix Goncourt du Premier Roman. Nous voici donc face à trois de multiples exemples paradigmatiques de ces intellectuels qui, venant d'ailleurs, ont choisi la France comme terre d'accueil et la langue française comme moyen d'expression.

La littérature francophone devient, par conséquent, un archipel de création artistique qui relève le surgissement de ce nouveau paradigme. C'est dans ce contexte qu'Anne-Rosine Delbart présente en 2005 un bilan sur ce corpus d'écrivains venus d'ailleurs sous le titre : Les exilés du langage. Un siècle d'écrivains français venus d'ailleurs (1919-2000). Suivant cette approche théorique sur les écrivains allophones d'expression française, Véronique Porra publie en 2011 Langue française, langue d'adoption. Une littérature "invitée », entre création, stratégies et contraintes (1946-2000). Le tournant du siècle a également permis de repenser le scénario littéraire francophone et c'est en 2012 qu'Ursula Mathis-Möser et Birgitz Mertz-Baumgartner présentent Passages et ancrages. Dictionnaire des écrivains migrants de la langue française (1981-2011). La même année, Joanna Nowicki et Catherine Mayaux s'attardent sur L'autre francophonie, un ouvrage qui prétend parler et faire parler de ces écrivains francophones venus de cette Europe que l'on appelait de l'Est. Nous ne pouvons pas clore ce brassage terminologique, sans citer Xénographies féminines dans l'Europe d'aujourd'hui (Alfaro, Sawas et Soto 2020), un ouvrage collectif récemment publié aux éditions Peter Lang qui propose une réflexion sur l'intérêt et la pertinence de travailler sur un corpus d'écrivains venus d'ailleurs ainsi que « de souligner, au croisement des sciences humaines et sociales, la nécessité d'une prise de conscience convoquant les parcours de la reconnaissance, pour reprendre l'expression de Paul Ricœur » (Alfaro, Sawas et Soto $2020: 11$ ).

À cet égard, il nous semble nécessaire de signaler notre préférence pour le terme de xénographies francophones dans l'objectif de dessiner la nouvelle cartographie littéraire dans le domaine qui nous occupe. En abordant les xénographies francophones en Europe, nous faisons référence à un espace géopolitique multiple et pluriel qui expose la représentation discursive d'une mosaïque littéraire qui met en musique " a space in which a new paradigm of plural identity is constructed. This is literature written hors-lieu [off site], characterized by the existentialist project of constructing a new personal identity » (Alfaro 2016: 233). Cette littérature illustre une dialectique 
entre l'espace national français, où cette littérature est publiée, et l'espace littéraire francophone, que ses écrivains occupent. De même, ces écrits proposent une dialectique entre le sujet migrant, représenté aussi bien à la première personne du singulier -le moi, écrivain- que du pluriel -le nous, collectif migrant-, et le sujet autochtone, représentant l'altérité aussi bien d'un point de vue individuel que collectif. Cette pléiade d'artistes, qui permet de penser l'expérience migratoire sous une multiplicité de perspectives, se sert du parcours personnel pour prôner les valeurs inclusives au sein des sociétés contemporaines.

Cette notion reçoit, en outre, un éclairage particulièrement intéressant lorsqu'elle s'attarde sur l'analyse d'un corpus d'auteures, car ces textes expriment une nouvelle modernité au féminin qui propose « une réflexion intellectuelle et esthétique, des cadres novateurs qui puissent tracer les voies où les conditions sociales seraient favorables à tous, hommes et femmes » (Alfaro, Sawas et Soto 2020 : 233). C'est dans ce contexte que nous convoquons la figure de la romancière Hadia Decharrière qui, née à Koweït en 1979 et ayant été élevée entre la Syrie, la France et les États-Unis, vit une expérience territoriale multiple et fait, par conséquent, partie de ces écrivains pour qui le voyage est devenu un moyen de configuration identitaire. Les effets de ce processus de brouillage transfrontalier se font écho dans un parcours littéraire construit en langue française qui met en musique les enjeux identitaires liés à l'expérience du déplacement.

Le voyage s'érige ainsi, au sens figuré, en palimpseste identitaire dans l'univers narratif décharrièrien. À cet égard, il convient de signaler que nous ne nous attacherons pas à décrire le principe de palimpseste dans le procédé d'écriture suivant la théorie de Gérard Genette (1982). Nous reviendrons, néanmoins, sur le sens originaire de ce terme d'origine latine désignant ce qu' " on gratte pour écrire de nouveau » (Gauvin, Van den Avenne, Corinus et Selao 2013 : 7). Nous analyserons, par conséquent, les différentes couches de l'expérience existentielle exprimées par l'auteure tout au long de l'aventure romanesque. C'est ainsi que nous nous attacherons de penser le processus de définition identitaire en métaphore de ce "parchemin dont on a gratté la première inscription pour en tracer une autre, qui ne la cache pas tout à fait, en sorte qu'on peut y lire, par transparence, l'ancien sous le nouveau » (Genette 1982 : quatrième de couverture). La mobilité trace, de ce fait, les lieux de mémoire d'une expérience autofictive où auteure et personnage illustrent le socle des identités multiples. L'objectif est donc d'analyser la restitution d'un vécu bioromancé où la construction identitaire devient la métaphore de ce « palimpseste [qui] reprend la trace, l'écho, l'empreinte, le projet d'un autre [...] [vécu] pour le déconstruire, le reconstruire, le critiquer » (Gauvin, Van den Avenne, Cornus et Selao 2013 : 7). De ce fait, nous pouvons affirmer que si « por síndoque el palimpsesto lo que está proclamando es cómo su condición de pluralidad textual es la condición de todos los textos aun cuando no se configuren como palimpsestos » (Blesa 2012: 214), suivant cette même métaphore le voyage evoque la source d'un palimpseste identitaire construit à travers la pluralité des voix qui configurent le parcours existentiel des sujets migrants.

Il convient de signaler à ce stade de la réflexion, qu'Hadia Decharrière illustre dans son parcours romanesque un exemple paradigmatique de ces auteures qui par- 
sèment l'édifice autofictif des lieux de l'extrême contemporain (Obergöker 2011). Le voyage et l'expérience de déracinement configurent la quête identitaire exposée aussi bien dans Grande Section, son premier roman, que dans Arabe, son second roman. Si le voyage devient une expérience réelle de déterritorialisation dans Grande section-le roman ici étudié- il est intéressant de noter que la protagoniste d'Arabe entreprend, de même, un voyage intérieur suite à la découverte soudaine de la langue arabe. Nous pouvons affirmer, par conséquent, que les deux axes thématiques qui articulent notre analyse représentent le sceau d'une écriture à portée autofictive. Il est donc nécessaire de tracer l'expérience bioromancé de l'écrivaine dans un premier temps pour mieux appréhender la manière de gérer et de penser l'identité dans l'écriture de l'intime (Lejeune 2015 : 104). Nous tenterons, par la suite, d'analyser la manière dont la topographie transfrontalière et la rencontre avec l'altérité tracent des empreintes indéniables sur les premières inscriptions d'un socle identitaire désormais multiple et pluriel. Cette réflexion nous permettra de mieux appréhender la manière dont ce roman protéiforme met en exergue la sensibilité aux identités multiples et, par conséquent, à une vision sociale humaniste.

\section{Hadia Decharrière, une traversée transfrontalière}

Il suffit de la googler et de tomber sur son profil Doctolib pour se rendre à l'évidence : Hadia Decharrière-Hamzawi avait peu de chance d'échapper plus long temps à son gène créatif. Une sœur humoriste que vous connaissez bien, un frère scénariste dont vous avez sans doute vu la comédie 20 ans d'écart: dans la famille, devenir chirurgien-dentiste relevait de l'accident industriel (Kopiejwski 2017 : en ligne).

Voici les propos de Cheek Magazine pour commencer à élaborer le profil de cette femme qui, à l'occasion des 30 ans de la mort de son père, décide d'entreprendre le chemin de l'écriture. Chirurgien-dentiste consacrée, Hadia Decharrière fait partie de ces écrivains en herbe qui parsèment les étals de librairies de l'extrême contemporain. Tout comme bon nombre de ses pairs, cette auteure se laisse emporter par l'aventure romanesque en parallèle à son activité professionnelle principale. Ne s'agissant pas d'un fait isolé, nous pouvons constater que cet avènement à l'écriture met en évidence un changement de paradigme du statut même de l'écrivain à l'époque actuelle. Désormais changeantes et prolifiques, les sociétés contemporaines germent un profil d'écrivain à temps partiel qui se permet de jongler entre l'écriture et d'autres activités professionnelles. L'écrivain devient, par conséquent, une figure de l'entredeux. La pléiade d'artistes qui épousent l'écriture et d'autres métiers est longue, nous pouvons citer, par exemple, Lætitia Colombani qui est réalisatrice, actrice, scénariste et romancière, mais aussi Maryam Madjidi qui enseigne le Français langue étrangère à la Croix Rouge et se consacre également à l'écriture, ou encore Rouja Lazarova qui, journaliste indépendante, commence à écrire en français d'abord des articles de presse, puis de la littérature. 
C'est dans ce contexte qu'Hadia Decharrière, née Hadia Hamzawi, commence un voyage dans le temps et l'espace des souvenirs pour puiser des sources littéraires qui donneront vie à son premier roman. Le discours narratif lui permet d'établir un dialogue entre ses souvenirs d'enfance et sa vie d'adulte, entre les voyages qui ont forgé son socle identitaire et son intégration dans le socle identitaire Français. L'auteure commence de ce fait un parcours proche de l'errance dans sa recherche de l'inaugural où l'identité évoque « une sorte de foyer virtuel » (Lévi-Strauss 1987 : 332) composé d'éclats de vie d'ici et de là, de la France et de l'ailleurs. La mémoire conforme ainsi le sol identitaire d'une construction romanesque où l'auteure, tout comme bon nombre d'écrivains contemporains, se montre "réticent[e] à écrire des pures et simples fictions " (Hubier 2003 : 115) et, de ce fait, son roman acquiert une valeur double «à la fois littéraire et documentaire » (Holtz et Masse 2012 : 11). Le corpus des xénographies francophones contemporaines illustre, par ailleurs, ce vif et croissant intérêt pour l'autofiction, ce néologisme créé par Serge Doubrovsky en 1975 (Grell 2014 : 8) que certains définissent comme une invention postmoderne (Zuffrerey $2012: 5$ ) qui permet « de distinguer la sensibilité moderne de la sensibilité classique » (Doubrovsky, cité par Jeannelle et Viollet 2007 : 65). Nous pouvons affirmer, par conséquent, que Grande section est une production autofictive tout à fait exemplaire de la démarche des nouveaux romanciers qui évoquent « la valeur du témoignage [et] du document 》 (Fosalau 2012: 220) de l'expérience vécue. C'est ainsi que le roman ici analysé présente une prédilection esthétique pour l'introspection à travers le récit fragmentaire d'une enfance rythmée par les allers-retours familiaux.

En effet, marquée par la Guerre froide, la famille Hamzawi quitte la Syrie pour s'installer aux États-Unis, un voyage qui changera à tout jamais la vie de cette lignée, car le père y perdra sa vie à cause d'un cancer. La mort de la figure paternelle illustre le point de départ d'un édifice narratif où l'auteure explore avec maîtrise son parcours personnel. Le voyage s'érige ainsi en palimpseste identitaire car c'est à travers un voyage dans le temps des souvenirs que l'auteure retrace un voyage initiatique qui tisse le parcours identitaire de cette jeune fille devenue adulte. La réflexion identitaire devient ainsi l'axe principal d'un roman divisé en trois parties d'extension inégales et intitulées : Premier trimestre, Période syrienne et réminiscences cannoises ; Deuxième trimestre, Parenthèse américaine ; Troisième trimestre, Absence et Permanence. Le temps écoulé le long des pages ne se correspond pas, néanmoins, à ces trois trimestres qui divisent l'année scolaire de la grande section. Il s'agit de retracer une dialectique entre le temps passé et le temps présent où la grande section fait aussi bien référence à l'enfance de l'auteure qu'à l'enfance de sa fille. Le roman se termine, par ailleurs, sur un épilogue qui, situé à Paris le 29 mai 2015, symbolise l'envol de cette romancière en herbe qui, ouvrant une enveloppe, retrouve un billet d'avion pour Los Angeles. La circularité romanesque évoquée à travers le voyage illustre la métaphore du palimpseste identitaire. Retourner à Los Angeles symbolise l'acceptation aussi bien de son identité multiple que de la perte de la figure paternelle.

Si Cannes, Damas et San Diego dessinent le scénario de cette traversée bioromancée, Paris sera l'espace qui accueille son deuxième roman, Arabe. Le récit met en scène une 
jeune Cannoise appellée Maya et installée à Paris qui se réveille un jour sachant parler la langue arabe. Voici le point de départ d'un roman qui s'écoule le long d'une journée où la protagoniste déambule aussi bien sur la ville de Paris que sur ses pensées. La langue devient alors pour Maya une source de réflexion qui traverse les frontières graphiques, phonétiques, syntaxiques et lexicales pour aller au-delà dans la construction identitaire comprise comme acteur social.

La réflexion identitaire et l'expérience personnelle se situent ainsi au cœur de l'édifice romanesque decharrièrien. S'inspirant de son propre vécu, Hadia Decharrière nous propose de penser la pluralité intrinsèque aux identités multiples qui configurent le socle des sociétés contemporaines. Le voyage se présente alors sous un prisme multiple qui imbrique le déplacement physique et psychique, la reconfiguration identitaire et la création d'une nouvelle dynamique de la dualité. Les regards portés sur l'identité dans ces deux romans symbolisent un parcours de reconnaissance vers les identités multiples qui prône l'avènement d'un nouveau paradigme social basé sur la tolérance et l'inclusion.

\section{Grande Section, le voyage comme palimpseste identitaire}

C'est dans ce contexte que nous présentons une approche analytique de Grande Section, le premier roman d'Hadia Decharrière paru en 2017 aux éditions J. C. Lattès. Ce roman s'érige en traversée d'une identité forgée à travers les différentes escales de son enfance. C'est ainsi que, née au Koweït -pays qu'elle quittera l'année de sa naissance pour le sud de la France-, l'auteure trace son parcours romanesque à travers une écriture sérielle rythmée par les souvenirs d'une enfance située géographiquement entre le Moyen-Orient, l'Amérique et l'Europe. L'auteure se sert du discours autofictif comme moyen pour partager son expérience personnelle sous la toile de la fiction littéraire ; autrement dit, l'auteure fouille dans l'archéologie mémorielle pour rêvetir la paratopie identitaire d'une rhétorique proche du palimpseste. En effet, si la première personne devient la projection de l'instance auctoriale dans l'univers narratif, le lecteur est néanmoins conscient de l'impossibilité de déterminer la véracité des épisodes qui sont évoqués dans le roman. Le pacte de lecture repose, par conséquent, sur une incertitude qui permet au lecteur de prendre plaisir dans l'expérience transfrontalière d'une lecture située à mi-chemin entre la fiction et la réalité et qui permet, en outre, " de désigner l'espace générique dans lequel se noue cette nouvelle relation dialectique entre écriture du moi et critique » (Gasparini 2011 : 14). L'écrivain, quant à lui, se sert des pratiques autofictives pour s'inventer " une personnalité ou une existence, tout en conservant son identité réelle » ou même « comme une pratique de vérité liée à l'écriture associative de la cure psychanalytique » (García 2009: 153).

Force est de constater à ce stade de la réflexion qu'Hadia Decharièrre plonge dans l'univers de la création littéraire d'une manière cathartique, suivant dans un premier temps les conseils de son psychanalyste pour affronter son deuil périnatal, puis pour répondre à un besoin personnel et à un vœu intérieur spontané. Elle ne s'oppose pas, néanmoins, à la possibilité de s'éloigner de l'aspect autofictif dans son parcours 
d'écrivain. C'est, en effet, dans ce contexte qu'elle déclare : « dans mon prochain livre, que je suis en train d'écrire, j'ai dépassé le côté psychanalytique, personnel et autocentré, et cela est passé par le fait de lâcher la première personne » (Kopiejwski 2017 : en ligne). Ce deuxième livre dont l'auteure parle n'est cependant pas Arabe, paru en 2019 aux éditions J. C. Latttès, mais un ouvrage resté pour l'instant inédit (Book Club 2019 : en ligne).

La toile de la représentation du roman ici analysé se lève comme suit :

Septembre. C'est la rentrée. Et, comme chaque année, une sensation d'excitation mêlée d'angoisse m'envahit, une anxiété bien particulière, spécifique à cette période, une douce mélancolie qui éclôt quand le temps devient maussade mais conserve la tiédeur estivale, et que les grandes vacances sont finies. J'ai le cafard, le cafard de la rentrée plus précisément ; c'est ainsi que ma petite sœur et moi avions baptisé ce sentiment ambivalent d'attraction et de répulsion scolaire qui s'invitait la dernière semaine d'août, pour ne nous quitter qu'aux vacances de la Toussaint. Nous avions autant d'envie d'y retourner que de nous débiner (Decharrière $2017: 13$ ).

La dynamique narrative suggère ainsi un récit qui, cultivant l'analepse et le portrait, met en musique les références spatio-temporelles intimement liées au monde de l'enfance pour évoquer la perception du monde qui émane de l'expérience vécue. Il est intéressant de noter que c'est à travers la circularité référentielle d'une rentrée symbolique que l'auteure se rapporte à l'un des lieux mythiques de l'enfance : l'école, un espace où d'année en année enfants et parents s'y retrouvent, les uns pour s'y rendre et les autres entant qu'accompagnateurs. L'usage d'une rhétorique proche de l'hypotypose éveille, en outre, les souvenirs d'enfance d'un lecteur qui voyage dans le temps suivant le rythme des phrases. C'est ainsi que mot à mot le lecteur se retrouve face au matériel scolaire, aux camarades de classe et même face au "savon jaune accroché au mur qui tourne sur lui-même » (Decharrière 2017 : 15), une réalité, par ailleurs, bien française. De ce fait, l'école symbolise le point de départ d'un voyage transfrontalier qui permet de penser le trajet non seulement du point de vue du déplacement physique mais aussi comme traversée de l'intime évoquant, au sens figuré, les traces du palimpseste identitaire. La première frontière est ainsi établie dans ce récit rétrospectif qui met l'accent sur le rapport à l'espace d'une jeune fille qui se voit obligée, suivant les décisions parentales, de changer d'école, de maison ainsi que de pays dans une période vitale où l'individu bâtit les ciments de sa construction identitaire. Face donc à la sécurité des lieux connus qui rassurent le personnage, l'école se caractérise par cette topographie changeante où la rentrée évoque une dimension spatio-temporelle multiple liée au déplacement.

La rentrée devient également un espace de dialogue entre la protagoniste et sa fille, dont les questions parcourent le discours narratif sous forme de leitmotiv tout le long du roman. La gallerie des portraits se multiplie tout au long de ce roman qui prend la forme d'un monologue protéiforme étayant le rapport entre le passé et le présent de la 
narratrice, protagoniste et auteure ; autrement dit, de la triade autofictive. À cet égard, il est important de s'attarder sur le regard que la mère porte vers son enfant :

Quand je la regarde aujourd'hui, je me rappelle mes cinq ans, ma grande section, et une irrépressible envie de pleurer m'envahit, pleurer pour de vrai avec des sanglots, le hoquet, et les taches rouges qui envahissent le visage, pleurer tant cette année fut chaotique ; je me croyais si grande, quand je ne l'étais tellement pas. En pleine possession de sa vie elle se sent grande, elle sait avoir une longueur d'avance, profiter de tout, ne s'inquiéter de rien, sentir la liberté, [...] décider et assumer, s'entêter même quand on sait qu'on a tort, tenir bon, s'acharner, puis, à un moment, lâcher. C'est peut-être ça que je n'ai jamais su faire moi, lâcher. Mais moi je n'ai pas choisi d'être grande à cinq ans [...] j'aurais voulu rester une enfant, avec l'innocence et l'insouciance que cela implique, ignorer tout de la maladie et de la séparation (Decharrière $2017: 21-22$ ).

Éminemment poétique, le statut de l'enfant dans l'économie du texte évoque l'ancrage affectif comme un élément déterminant dans le processus de construction identitaire de tout individu. La dialectique est ainsi présentée sous la représentation de l'enfance de la mère et de la fille, entre le passé et le présent, entre la souffrance et l'insouciance. En somme, il s'agit de mettre en musique le regard introspectif de la narratrice adulte pour retracer les différentes couches d'un palimpseste identitaire dont les traces témoignent de la mouvance topographique de l'auteure.

Le travail mémoriel de l'instance narrative entreprend ainsi le chemin des souvenirs pour traverser les frontières du cadre spatio-temporel du présent. Suite aux différents déménagements, la rencontre interculturelle se révèle fondamentale dans la construction identitaire du personnage. Dans ce contexte, la première destination s'avère être la Syrie, « pays des anciens, des grands-parents, un pays où l'on naît et ou l'on meurt mais dans lequel on ne vit pas » (Decharrière 2017 : 28). Un pays où en 1984 les magasins d'alimentation sont « aussi rachitiques que le sont leurs homologues soviétiques » (Decharrière 2017 : 30). La quête identitaire se déclenche ainsi dans le fort intérieur d'une jeune fille qui doit s'adapter aux différents espaces géographiques qui traceront le palimpseste de son socle identitaire, car « el lugar es elemento fundamental de toda identidad, en tanto que autopercepción de la territorialidad y del espacio personal » (Aínsa $2006: 22$ ).

C'est alors que, vivant à Damas, la protagoniste perd même l'un des traits constituants son identité, son prénom :

Mes grands-parents ne m'appellent pas par mon prénom, mais par la position familiale qui les lie à moi, je suis le témoin de leur positionnement dans l'arbre généalogique, un miroir de l'emplacement de chacun de mes aïeux. Comme Fantômas j'emprunte une de mes multiples identités en fonction de mon interlocuteur, dès lors qu'on me parle en arabe, j'adopte celle de celui ou celle qui me fait face (Decharrière 2017 : 79). 
À cet égard, il convient de signaler que chaque culture a une pratique onomastique qui lui est propre. Ce passage met en lumière la différence existante entre la Syrie et la France en matière d'identification à travers les noms et les prénoms. Si d'après la narratrice à Damas les rapports familiaux s'établissent par rapport à la lignée, en Europe occidentale l'habitude veut que le prénom soit un référent qui relève du choix parental dès la naissance. Le legs onomastique s'érige alors en porteur d'identité non seulement d'un point de vue généalogique à travers le patronyme, mais aussi d'un point de vue individuel qui révèle des données fondamentales telles que la langue et la culture d'origine, entre autres. Nous sommes face à deux approches différentes sur la question identitaire qui convergent dans une pratique commune : le prénom permet de se distinguer de l'autre, de s'affirmer entant que moi et de profiler une identité singulière au sein d'une communauté. C'est ainsi que nous pouvons affirmer que le nom symbolise l'un des traits identitaires essentiels à l'individu. Le constat de ce changement en fonction du contexte s'avère être l'évidence d'une identité multiple et, par conséquent, source de questionnement identitaire.

La question qui se pose dans l'expérience itinérante est, comment se définir au sein de la société que l'on habite? Il vaut la peine de s'attarder sur les enjeux identitaires des appartenances multiples pour mieux comprendre l'aspect pluriel et parfois paradoxal de leur socle identitaire. C'est dans cette perspective que la narratrice n'hésite pas à affirmer l'imprécis de son ancrage identitaire :

Parce que mes parents sont syriens, parce que j'au vécu en France de ma naissance à mes quatre ans, parce que malgré tout je ne suis pas française, parce qu'avec cet accent san diegois que j'ai chopé pendant la parenthèse américaine les Californiens me prennent pour une de leurs. Alors, ça devient toujours très compliqué quand les gens me demandent d'où je viens. Je ne peux pas dire Je suis française car c'est incomplet, ni Je suis syrienne parce que c'est faux, alors j'ai ma phrase toute faite que je prononce d'une profonde inspiration Je suis française d'origine syrienne mais je suis arrivée en France à l'âge de deux semaines donc c'est comme si j'étais française en fait. Et à mon grand dam, on me renvoie systématiquement. Ah, tu es née en Syrie? qui me contraint à me lancer dans des explications qui ne font que mettre en lumière les grandes complications de mon enfance (Decharrière 2017 : 36-37).

Relever les défis identitaires posés dès son enfance devient, pour Hadia Decharrière, un voyage vers ce passé qu'elle ne pourra jamais effacer. La lecture nous submerge, en effet, dans l'expérience d'un déracinement identitaire sous le prisme du quotidien. À la fois d'ici et d'ailleurs, ce personnage illustre une « problématique qui met en scène ce qui se joue dans la relation avec l'Autre et ouvre ainsi une véritable réflexion sur l'altérité en même temps qu'il posera la question des origines et de la perte des origines » (Albert $2005: 17$ ). Autrement dit, le socle identitaire des hommes dépaysés (Todorov 1996) se présente sous le prisme d'un dialogue interculturel perpétuel où le rapport aux différentes cultures et aux différentes langues s'entrelacent pour relever le défi de la construction identitaire. L'édifice existentiel tresse ainsi le 
legs du déracinement pour regrouper les différents éclats de vie d'ici et d'ailleurs dans la mosaïque identitaire.

Située au troisième chapitre, la citation que nous venons d'évoquer met en évidence l'importance accordée à la construction personnelle. De ce fait, il convient de s'arrêter un instant pour réfléchir autour du concept d'identité. Si dans les dictionnaires ce terme propose des acceptions qui convergent dans l'unité des traits constitutifs des individus, l'évolution épistémologique de ce terme a longuement évoluée. En effet, au XIX ${ }^{\mathrm{e}}$ ce terme relevait du domaine de la médecine légale dans la mesure où il renvoyait « à la reconnaissance d'une personne en état d'arrestation, d'un prisonnier évadé, d'un cadavre ou squelette soumis à l'examen des services de police judiciaire » (Detienne 2010 : 141). Puis, cette notion venait accompagner la création de la carte d'identité. De nos jours, d'une manière consensuelle, la carte d'identité présente les données essentielles pour identifier un individu, c'est-à-dire : une photo, la date et le lieu de naissance, l'adresse postale et l'empreinte digitale, puis la date de validité du document d'identification. Il est évident que ces informations sont accompagnées d'autres jugées nécessaires selon les différents pays. La réflexion qui découle de cette évolution technique du terme nous invite à parler d'identité comme la base qui défini un individu, mais aussi comme une quête de singularité au sein même d'un collectif avec lequel l'individu partage les mêmes codes, les mêmes traits et les mêmes référents culturels.

À ce stade de la réflexion, il convient de rappeler que les circonstances qui déterminent le départ, marquent, en outre, l'expérience de déterritorialisation. La première destination de cette jeune fille est une ville où « les grilles d'aération des sous-sols de boulangeries ne dégagent pas de bonne odeur de pâte à croissant, et les kiosques ne vendent pas leurs premiers journaux » (Decharrière 2017 : 53). L'odorat relève ainsi d'une importance déterminante dans la construction identitaire de la narratrice. Et, dans ce contexte, il est fort intéressant de signaler le rôle octroyé aux boulangeries françaises, et à la gastronomie en général, dans les textes appartenant aux xénographies francophones de l'extrême-contemporain. Suite à bon nombre de lectures, nous avons pu constater que la gastronomie symbolise le plus souvent un élément de réflexion dans le processus de construction identitaire. Pour Hadia Decharrière, tout comme pour Maryam Madjidi (Marx et la poupée, 2017), la boulangerie devient l'étendard de la culture gastronomique française. Si pour la petite Maryam à son arrivée en France le croissant est porteur d'une altérité imposée, pour Hadia le parfum des boulangeries, tel la madeleine pour Proust, éveillera la mémoire involontaire qui lui évoque son rapport identitaire à la France.

La gastronomie s'érige, de même, en symbole identitaire à San Diego, deuxième escale :

La nourriture est globalement assez étrange ici, on dirait que l'aspect prime sur le goût et la texture. Les glaces turquoise au bubble-gum reproduisent parfaitement le goût tutti-frutty des billes à mâcher sans en avoir la fonction essentielle, les peanut butter and jelly sandwichs, deux toasts comprimant de la gelée rose flou fricotant 
avec du beurre de cacahuète, calent les appétits les plus féroces [...]. Ils mettent du cheese partout, littéralement du fromage, en vrai une espèce de pâte jaune fluorescente qu'on achète en tube, comme de la mayonnaise (Decharrière 2017 : 105).

La gastronomie et les bonnes manières à table sont de toute évidence propres à chaque culture et, de ce fait, c'est un contexte qui évoque aussi bien l'ensemble de traits culturels propres à un groupe ethnique que le sentiment d'appartenance d'un individu à un groupe (Beniamino et Gauvin 2005 : 96). La référence culturelle associée à la gastronomie autochtone évoque, de ce fait, non seulement la culture d'accueil, mais aussi ses origines étrangères. Les référents culturels diffèrent, en effet, que l'on se trouve dans un pays ou dans un autre et même si l'on parle d'un même produit celui-ci est évoqué dans l'imaginaire collectif en fonction de sa réalité représentative.

Dans ce contexte, le départ pour les États-Unis se présente comme une expérience traumatisante pour une jeune fille qui se voit obligée de quitter la Syrie :

Comme pour des vacances, [...] pendant les vacances de février [...]. À peine l'avion avait-il atterri en France que des gens vinrent chercher mon frère et ma sœur aînés à Roissy, dans le terminal des arrivées. Personne ne disait rien, les adultes en imperméables beiges restèrent muets tout au long de la transaction. Puis, avec mon père, ma mère et ma petite sœur, nous partîmes présenter nos passeports à un comptoir Air France. [...] Cette fois, nous volâmes beaucoup plus longtemps, des heures et des heures, peut-être des jours entiers, un temps infini, [...] puis nous arrivâmes à Los Angeles.

Je sortis de l'avion totalement déboussolée, intimidée par tant d'inconnu (Decharrière 2017 : 93-94).

Ce moment est raconté à travers les yeux d'une enfant qui ne comprend ni le départ ni la séparation familiale. La question de la langue devient un point essentiel de réflexion qui marque la géopoétique romanesque. Logée chez ses oncles, la famille doit s'adapter à un nouveau contexte et à une nouvelle langue. C'est ainsi que la narratrice affirme avoir expérimenté un " très grand souci de l'identique dans cette Amérique adolescente » (Decharrière 2017 : 98) et un souci d'appartenance langagière. Aux ÉtatsUnis la langue pose ainsi un problème à cette jeune fille qui doit interagir avec ses cousines. Il est fort intéressant de noter à ce stade de l'analyse la réflexion émannant des relations interpersonnelles avec sa cousine : « on ne parle pas la même langue mais ça ne nous empêche pas de nous disputer tout le temps. La discorde a sans doute son propre dialecte, une langue universelle, celle qu'empruntent les pays aux langues officielles différentes lorsqu'ils entrent en guerre » (Decharrière 2017 : 100). L'affirmation identitaire face à l'altérité se voit ainsi empreinte d'un affrontement hostile, métaphore du conflit identitaire des individus vivant dans l'entre-deux.

Le processus de construction identitaire de la narratrice se construit, par ailleurs, sous la différence représentative du moi et de l'autre à travers la comparaison entre 
l'identité de la protagoniste et l'altérité que représente sa cousine. C'est ainsi que la jeune fille se sent identifiée à une langue qui n'est pas la langue du pays d'accueil et, par conséquent, elle fait du français son étendard identitaire. Elle affirme, en effet, « on ne voit pas le monde de la même façon, et chacune ressent le besoin d'imposer son point de vue sans considérer les affects de l'autre. Quand elle essaye de me convaincre qu'un $i$ s'écrit "E" j'ai envie de lui mettre un poing, et je lui explique agacée que c'est un [euh] son truc, pas un $i »$ (Decharrière $2017: 100-101$ ).

La parenthèse américaine symbolise un nouveau point de départ pour cette famille qui doit faire face à la perte de la figure paternelle. À l'expérience de déracinement, de diglossie et d'hybridation interculturelle, vient se superposer le deuil et la reconfiguration familiale. Le parcours identitaire se voit ainsi complété par une appartenance religieuse : "Papa est un ange maintenant » (Decharrière 2017 : 110). La confession religieuse s'inscrit dans l'évolution identitaire de cette jeune fille qui, une fois devenue adulte, affirme ne pas croire en Dieu car " on perd tôt ou tard cette illusion de sécurité, dès lorsqu'on se retrouve confronté à l'idée de sa propre mort » (Decharrière 2017 : 111). La mort représente ainsi un voyage sans retour qui marque les lieux de la mémoire de cette jeune fille qui remémore l'évolution de la maladie dans un espace clos, sombre et inaccessible où le père, figé, attendait la fin de ses jours. Force est de constater à ce stade de l'approche analytique que « las relaciones con el pasado no son nunca neutras y se inscriben inevitablemente en la más compleja dialéctica que hacen de su reconstrucción una forma de la memoria, cuando no de la nostalgia y de la fuga desencantada del presente hacia el pasado » (Aínsa 2006 : 136). L'expérience autofictive se penche ainsi sur le rapport à la mort et à l'expérience du deuil comme ce départ qui, attendu ou innatendu, laisse un vide existentiel et une empreinte dans ce palimpseste identitaire construit sur le chemin de la vie.

Si la deuxième partie de l'édifice romanesque se termine sur la fin de la vie, la troisième partie, intitulée Absence et Permanence, commence avec l'arrivée d'un nouveau venu dans la famille. Ce nouveau départ est ainsi évoquée grâce à l'adoption d'un lapin pour Maya, la fille de la protagoniste. Le lecteur ouvre ainsi un chapitre également penché sur un va-et-vient spatio-temporel où la narratrice revient sur la question des origines :

Je parle anglais, plutôt bien [...]. J'en ai l'air mais qu'on ne se fie pas aux apparences, je ne suis pas américaine, je ne le serai jamais. Je ne serai jamais syrienne non plus, [...]. Mais, ce que je ne suis pas encore en mesure d'admettre, le serai-je un jour ?, c'est que, si je n'y suis pas, la Syrie, elle vit en moi, constamment, profondément, de façon inaltérable. [...] L'inné ne m'a pas quittée, je comprends les sons, les mots et les phrases, qu'on me parle en français, en anglais ou en arabe, mon oreille conserve son aptitude ; mais je ne parle pas, aucun mot arabe ne parvient à sortir de ma bouche, le conscient et le volontaire restent bloqués, quelque part entre l'acceptation de son identité et la fierté de ses origines. J'ai cessé d'assumer mon trilinguisme quand mon père à cesser d'exister (Decharrière $2017: 169$ ). 
La réflexion intrinsèque au sentiment d'appartennance lié à l'expérience langagière devient ainsi le reflet de ce deuil et fait partie du parcours identitaire de l'auteure. La langue devient, par conséquent, l'un des traits identitaires indispensables à la construction personnelle et la littérature retrace ce parcours quotidien qui permet d'aborder la société à partir de l'expérience autofictive. L'aspect linguistique dans la traversée romanesque decharrièrienne devient d'autant plus important qu'elle le situe au cœur de son deuxième roman. Vivant une situation de xénoglossie, la protagoniste se réveille un jour parlant l'arabe, une langue en principe inconnue de son entourage. La figure paternelle s'avère être le lien entre la langue arabe et une protagoniste qui s'avère être homonyme de la fille de la narratrice. Est-ce un clin d'œil à la transmission des origines et, par conséquent, de la quête identitaire ? Seraitce l'itinéraire héréditaire d'un palimpseste identitaire tracé au fil des voyages ? Loin d'avoir une réponse à ces questions, nous nous permettons de parler et de faire parler d'un destin individuel qui évoque les retrouvailles subites avec une langue porteuse d'histoire et illustrant ainsi non seulement l'éveil de la langue de ses aïeux, mais aussi l'acceptation de son appartenance multiple.

Nous pouvons affirmer, par conséquent, que la langue française est mise à l'honneur dans le projet scriptural de cette écrivaine qui se sert de la littérature pour créer un lieu de rencontre et du voyage pour tracer son palimpseste identitaire. Cet espace d'apprentissage met en exergue les références culturelles associées à son identité. Et, c'est dans un tel contexte d'immigration que se dessinent en partie les réalités caractéristiques du socle identitaire. L'édifice romanesque decharrièrien met en lumière l'illusion forgée à travers la réalité langagière des personnages vivant une expérience de déracinement où les concepts d'altérité et d'identité se fondent dans le for intérieur d'une identité désormais interculturelle. La construction existentielle est, en conséquence, intimement liée à la manière d'appréhender le monde, de tracer et de retracer l'expérience personnelle, d'écrire le parcours de l'intime et de présenter les empreintes du voyage dans un palimpseste identitaire.

\section{Conclusion}

Il importe, au moment de conclure, de souligner que le projet scriptural d'Hadia Decharrière reflète le processus de construction identitaire des nouveaux intervenants de la société, non seulement en France mais aussi ailleurs. Il est, effet, important de penser l'ancrage identitaire dans son rapport aux frontières geopolitiques ainsi qu'au voyage personnel comme une source de stimulation sensorielle et intellectuelle, individuelle et collective. La littérature permet dans ce contexte de peaufiner et de mieux comprendre l'expérience de déracinement des identités multiples à travers l'approche autofictive et s'inscrit ainsi dans l'ère actuelle qui confirme " el renovado interés por el destino individual en el seno de un devenir histórico común [y que] también explica el sentimiento de la existencia de un tiempo individual en la representación del tiempo colectivo compartido en un espacio común » (Aínsa 2006 : 136). L'espace de création littéraire devient, par conséquent, l'un des seuils permettant d'aborder le changement 
de paradigme de sociétés futures. Ce qui s'imbrique dans le discours de Federico Mayor Zaragoza (Alfaro, Arias, Gamba 2019: 10) lors qu'il prône :

Es necesaria una gran movilización que permita a todos los seres humanos ser conscientes de la situación presente y, en consecuencia, comprometerse a actuar sin demora con un comportamiento cotidiano apropiado que haga posible hacer frente a procesos potencialmente irreversibles. Las comunidades académica, científica, artística, literaria, intelectual en suma, deben liderar hoy las respuestas adecuadas a las amenazas que se ciernen sobre la humanidad a escala mundial.

La démarche volontaire vers la langue française s'érige, en outre, en caractéristique inhérente à ce corpus d'écrivains appartenant au domaine des xénographies francophones de l'extrême contemporain et mettant cette problématique au sommet de l'iceberg identitaire. La traversée personnelle illustre une opportunité de reconfiguration identitaire et situe à ces agents de la société en médiateurs culturels et, par conséquent, en passeurs de frontières. Le phénomène du déracinement affecte l'écrivain, mais aussi la société d'accueil. La littérature peut alors devenir un pont symbolique entre l'individu et la société qui dépasse les frontières géopolitiques traditionnelles et prône le dialogue interculturel.

La littérature dessine, par conséquent, une nouvelle géopoétique où des prénoms aux goûts d'ailleurs parsèment un espace transnational européen qui évoque une " géo-graphie singulière de l'histoire de l'Europe » (Alfaro 2013-2014 : 1260). Amin Maalouf, dont ses propos lèvent la toile de notre analyse, et Hadia Decharrière s'érigent ainsi en deux exemples emblématiques de ce changement de paradigme. Et, c'est dans ce contexte que nous nous permettons de reprendre la réflexion de Maria José Carneiro Dias (2011 : 122) pour penser le voyage comme palimpseste identitaire :

Voilà pourquoi la route et le voyage sont si importants dans [la] fiction. Souvent associé à l'apprentissage de soi et de l'autre, le voyage désigne, dans ce cas-là, un parcours existentiel. Expérience de déplacement physique, le voyage s'associe aussi à un déplacement de la perspective du regard et instaure, dans le mouvement qui lui est propre, un phénomène de différenciation entre le lieu premier et le lieu-autre de destination, avec une naturelle confrontation de référentiels, à travers laquelle le voyageur prend conscience de soi et se restructure lui-même sur de nouvelles plateformes relationnelles.

\section{BIBLIOGRAPHIE}

AINSA, Fernando (2006) : Del topos al logos. Propuestas de geopoética. Madrid : Iberoamericana.

ALBERT, Chirstiane (2005) : L'immigration dans le roman francophone contemporain. Paris : Karthala. 
ALFARO, Margarita (2016) : " Ectopic literature : the emergence of a new transnational literary space in Europe in the Works of Eva Almassy and Rouja Lazarova ». AVERIS, Kate et Isabel HOLLIS-TOURÉ (eds.), Exiles, travelers and vagabonds. Rethinking mobility in Francophone women's writing, Pays de Galles : University of Walles Press : 232-248.

(2013-2014) : « La construction d'un espace géo-poétique francophone en Europe : l'expérience totalitaire et la représentation de l'exil ». Revista portuguesa de literatura comparada, $\mathrm{n}^{\circ}$ 17-18, vol. II. Lisbonne : Dedalus, Ed. Cosmos: 1243-1260.

ALFARO, Margarita ; SAWAS, Stéphane et SOTO, Ana Belén (2020) : Xénographies féminines dans l'Europe d'aujourd'hui. Bruxelles : Peter Lang.

ALFARO, Margarita; ARIAS, Silvia et GAMBA, Ana (2019) : Agenda 2030: Claves para la transformación sostenible. Madrid : Los Libros de la Catarata.

APPADURAI, Arjun (1996) : Modernity at large : Cultural Dimensions of Globalization. Minneapolis : University of Minessota Press.

BENIAMINO, Michel ; GAUVIN, Lise (2005) : Vocabulaire des études francophones. Les concepts de base. Limoge : Pulim.

BLESA, Túa (2012) : « La escritura como palimpsesto (Una forma de la logofagia) ». Tropelías. Revista de Teoría de la Literatura y Literatura Comparada, $\mathrm{n}^{\circ} 18$, 204-215.

BOOK CLUB (2019) : Hadia Decharrière présente " Arabe » son deuxième roman. Disponible sur : https://www.youtube.com/watch?v=O0sgJCo07y0

CALEFATO, Patrizia (2018) : " Revêtir le voyage ». Sociétés, 2018/1 n o 39, 147-153.

CARNEIRO DIAS, Maria José (2011) : «Amin Maalouf : Le chemin vers l'autre se fait en voyageant. L'itinéraire comme stratégie de reconfiguration identitaire ». Intercâmbio, v. 4, 118-137.

DECHARRIÈRE, Hadia (2017) : Grande section. Paris : JC Lattès. (2019) : Arabe. Paris : JC Lattès.

DELBART, Anne-Rosine (2005) : Les exilés du langage. Un siècle d'écrivains français venus d'ailleurs (1919-2000). Limoges: Pulim.

DETIENNE, Marcel (2010) : L'identité nationale, une énigme. Paris : Gallimard.

FOSALAU, Liliana (2012) : « Espaces exiliaires - espaces identitaires chez trois écrivains francophones roumains ». Philologica Jassyensia, vol. 8 (2) : 211-221.

GARCÍA, Mar (2009) : «L'étiquette générique 'autofiction' : us et coutumes ». Çédille, $\mathrm{n}^{\circ} 5,146-163$.

GASPARINI, Philippe (2011) : Est-il je ?: Roman autobiographique et autofiction. Paris : Seuil.

GAUVIN, Lise ; VAN DEN AVENNE, Céciel ; CORNIUS, Véronique et SELAO, Ching (2013) : Littératures francophones. Parodies, pastiches, réécritures. Lyon: ENS Éditions.

GENETTE, Gérard (1982) : Palimpsestes. La littérature au second degré. Paris : Seuil. 
GRELL, Isabelle (2014) : L'autofiction. Paris : Armand Colin.

HOLTZ, Grégoire ; MASSE, Vincent (2012) : «Étudier les récits de voyage. Bilan, questionnements, enjeux ». Arborescences. Revue d'études françaises, $\mathrm{n}^{\circ} 2$, $1-30$.

HUBIER, Sébastien (2003) : Littératures intimes. Les expressions du moi, de l'autobiographie à l'autofiction. Paris : Armand Colin.

JEANNELLE, Jean-Louis ; VIOLLET, Catherine (2007) : Genèse et autofiction. Louvain-La-Neuve : Académia Bruylant.

KOPIEJWSKI, Faustine (2017) : " Avec "Grande Section", Hadia Decharrière publie un premier roman bouleversant ». CkEEk Magazine, publié le 7 avril 2017.

LEJEUNE, Philippe (2015) : Écrire sa vie. Du pacte au patrimoine autobiographique. Paris : Armand Colin.

LÉVI-STRAUSS, Claude (1987) : L'identité. Paris : PUF.

MAALOUF, Amin (2019) : Le naufrage des civilisations. Paris : Grasset.

MADJIDI, Maryam (2017) : Marx et la poupée. Paris : Le Nouvel Attila.

MATHIS MOSER, Ursula ; MERTZ-BAUMGARTNER, Birgitz (2012) : Passages et ancrages. Dictionnaire des écrivains migrants de la langue française (19812011). Paris : Honoré Champion.

NOWICKI, Joanne ; MAYAUX, Catherine (2012) : L'autre francophonie. Paris : Honoré Champion.

OBERGÖKER, Timo (2011) : Les lieux de l'extrême contemporain. Munich : Martin Meidenbauer.

PORRA, Véronique (2011) : Langue française, langue d'adoption. Une littérature "invitée », entre création, stratégies et contraintes (1946-2000). Hildesheum, Zürich, New York : Olms.

TODOROV, Tvetzan (1996) : L’homme dépaysé. Paris : Seuil.

TVERDOTA, György (199) : Écrire le voyage. Paris : Presses de la Sorbonne nouvelle.

WIHTOL DE WENDEN, Catherine (2013) : La question migratoire au XXIe siècle. Migrants, réfugiés et relations internationales. Paris : Presses de la Fondation Nationale des Sciences Politiques.

ZANGHI, Filippo (2014) : Zone indécise. Périphéries urbaines et voyage de proximité dans la littérature contemporaine. Lausane : Presses universitaires du Septentrion.

ZUFFREREY, Joël (2012) : L'autofiction : variations génériques et discursives. LouvainLa-Neuve : L'Harmattan-Academia.

\section{PERFIL ACADÉMICO-PROFESIONAL}

Doctora en Filología Francesa por la Universidad Autónoma de Madrid, Ana Belén Soto tiene una formación académica intercultural que le ha permitido enfocar su ámbito de investigación hacia el análisis de la literatura francesa y francófona contem- 
poránea. Se interesa especialmente por la experiencia del desarraigo lingüístico e identitario, por los fenómenos ligados a la desterritorialización y por la elección voluntaria del francés como lengua de escritura en un corpus literario escrito por mujeres. En la actualidad es Profesora Ayudante Doctora en la Universidad Autónoma de Madrid y es miembro del Grupo de Investigación ELITE (Estudios de Literaturas e Identidades Transnacionales en Europa).

Fecha de recepción: 07/04/2020

Fecha de aceptación: 29/06/2020 